\title{
ON SUPPRESSION OF CHAOTIC MOTIONS OF A PORTAL FRAME STRUCTURE UNDER NON-IDEAL LOADING USING A MAGNETO-RHEOLOGICAL DAMPER
}

\author{
Angelo Marcelo Tusset, Vinicius Piccirillo \\ Federal University of Technology-Paraná - UTFPR, Ponta Grossa, PR, Brazil \\ e-mail:tusset@utfpr.edu.br; piccirillo@utfpr.edu.br \\ José Manoel Balthazar \\ University Paulista State - UNESP, Rio Claro, SP, Brazil; e-mail: jmbaltha@rc.unesp.br \\ Reyolando Manoel Lopes Rebello da Fonseca Brasil \\ Federal University of ABC-UFABC, Santo Andre, SP, Brasil; e-mail: reyolando.brasil@ufabc.edu.br
}

\begin{abstract}
We consider chaotic motions of a portal frame structure under non-ideal loading. To suppress this chaotic behavior, a controlling scheme is implemented. The control strategy involves application of two control signals and nonlinear feedforward control to maintain a desired periodic orbit, and state feedback control to bring the system trajectory into the desired periodic orbit. Additionally, the control strategy includes an active magneto-rheological damper to actuate the system. The control force of the damper is a function of the voltage applied in the coil of the damper that is based on the force given by the controller.
\end{abstract}

Keywords: feedback control, feedforward control, MR damper

\section{Introduction}

The study of non-ideal vibrating systems, that is, when the excitation is influenced by the response of the system, has been considered a major challenge in theoretical and practical engineering research (as examples, among others, see Bolla et al. (2007), Castão et al. (2010), Samantaray et al. (2010), Djanan et al. (2013). In this work, we observed chaotic vibrations of a portal frame structural system mathematical model under non-ideal loading. We intend not only to suppress large amplitude oscillations but also to reduce them to a periodic orbit. The suppression of chaos and keeping the oscillations into a desired periodic orbit was obtained using active control considering the Optimal Linear Feedback (OLFC) proposed by Rafikov and Balthazar (2004) and the use of a magnetorheological (MR) damper modeled considering the hysteresis phenomenon.

The MR damper uses an MR fluid which is basically composed of micrometer-sized particles of iron suspended in an oil base. The MR response of MR fluids is a result of polarization induced in the suspended particles by application of an external field. The interaction between the resulting induced dipoles makes the particles form columnar structures parallel to the applied field, increasing the viscous characteristics of the device (Kasemi et al., 2012; Dutta and Chakraborty, 2014). These magnetic properties permit its use as a damper controlled by an electrical current (Tusset and Balthazar, 2013; Tusset et al., 2012, 2013). When using MR damper control for suppression of unwanted oscillations, the viscosity of the internal fluid varies according to a variable electrical current or voltage (Tusset et al., 2009; Piccirillo et al., 2014). According to Cetin et al. (2011), there are two main approaches in the literature to describe the hysteresis dynamic behavior of MR dampers. One of them is the Bouc-Wen hysteresis model proposed 
by Spencer et al. (1997), and other one is the LuGre hysteresis model that has been obtained from the nonlinear friction model proposed in (Jimenez and Alvarez, 2002, 2005; Terasawa et al., 2004; Sakai et al., 2003). In Dyke et al. (1996), the Bouc-Wen model of an MR damper was implemented with successful results to a three-story frame model. Cetin et al. (2011) used the LuGre friction model of an MR damper to a six-story frame model. Although Bouc-Wen model can accurately predict MR damper dynamics for active or semi-active control, it is too complex and difficult to implement (Cetin et al., 2011). On the other hand, Cetin et al. (2011) observed that the LuGre Model is more versatile than the Bouc-Wen model in semi-active control systems, therefore, in this work, the LuGre friction model is preferred over the Bouc-Wen model.

The organization of this paper is as follows: in Section 2, the mathematical model of the portal frame under a non-ideal excitation is described and numerical simulations necessary to analyze the dynamics of the system are performed. In Section 3, the control of chaotic motion by the application of the OLFC method is presented. Section 4 presents the mathematical model of the MR damper as a function of the applied voltage in its coil and numerical simulations necessary to analyze the dynamics of the controlled system. In Section 5, the robustness of the control techniques is tested by including parameters uncertainties on the control signals. The final remarks and the acknowledgments are in Sections 6 .

\section{Formulation of the engineering problem}

Here, we will consider the horizontal motion of a portal frame under a non-ideal excitation (see Fig. 1a) and the approximated schematic model of the system, represented by coupled oscillators (see Fig. 1b).
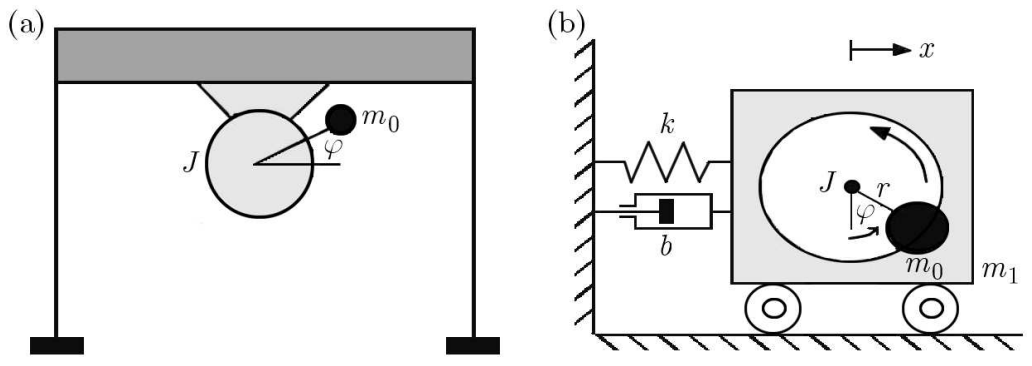

Fig. 1. (a) Non-ideal portal frame; (b) schematic of a non-ideal oscillator

The parameters of this coupled dynamical system consist of $m_{0}, m_{1}, k_{1}, k_{n l}, c_{1}, x_{1}, \varphi, J, r, d, s$, the mass, unbalanced mass, linear stiffness, non-linear stiffness, linear damping, displacement, angular displacement, inertia moment, eccentricity of the unbalanced mass. $d$ is related to the voltage applied across the armature of the DC motor and $s$ is a constant for each model of the DC motor considered. The resulting mathematical model of the structure is a Duffing-like equation

$$
\begin{aligned}
& \left(m_{1}+m_{0}\right) \ddot{x}+b \dot{x}-k_{l} x+k_{n l} x^{3}=m_{0} r\left(\ddot{\varphi} \sin \varphi+\dot{\varphi}^{2} \cos \varphi\right) \\
& \left(J+r^{2} m_{0}\right) \ddot{\varphi}-r m_{0} \ddot{x} \sin \varphi=L(\dot{\varphi})=d-s \dot{\varphi}
\end{aligned}
$$

Next, we render Eqs. (2.1) dimensionless in terms of new variables defined by: $\tau=\omega t, x_{1}=x / x^{*}$, and $x_{3}=\varphi / \varphi^{*}$, where $x^{*}$ and $\varphi^{*}$ are constant characteristics. Equations (2.1) can be represented in a state space, in dimensionless form, as

$$
\begin{array}{ll}
x_{1}^{\prime}=x_{2} & x_{2}^{\prime}=-\alpha x_{2}+\beta_{1} x_{1}-\beta_{3} x_{1}^{3}+\delta_{1} \sin \left(\varphi^{*} x_{3}\right) x_{4}^{\prime}+\delta_{1} \cos \left(\varphi^{*} x_{3}\right) x_{4}^{2} \\
x_{3}^{\prime}=x_{4} & x_{4}^{\prime}=\rho_{1} \sin \left(x_{3}\right) x_{2}^{\prime}-\rho_{3} x_{4}+\rho_{2}
\end{array}
$$


where

$$
\begin{aligned}
& \alpha=\frac{b}{\left(m_{1}+m_{0}\right) \omega} \\
& \omega=\sqrt{\frac{k_{1}}{m_{1}+m_{0}}} \\
& \beta_{1}=\frac{k_{l}}{\left(m_{1}+m_{0}\right) \omega^{2}} \\
& \beta_{3}=\frac{k_{n l} x^{* 2}}{\left(m_{1}+m_{0}\right) \omega^{2}} \\
& \delta_{1}=\frac{m_{0} r \varphi^{*}}{\left(m_{1}+m_{0}\right) x^{*}} \\
& \rho_{2}=\frac{d}{\left(J+r^{2} m_{0}\right) \omega^{2} \varphi^{*}} \\
& \delta_{2}=\frac{m_{0} r \varphi^{* 2}}{\left(m_{1}+m_{0}\right) x^{*}} \\
& \rho_{1}=\frac{r m_{0} x^{*}}{\left(J+r^{2} m_{0}\right) \varphi^{*}} \\
& \rho_{3}=\frac{s \omega \varphi^{*}}{\left(J+r^{2} m_{0}\right) \omega^{2} \varphi^{*}}
\end{aligned}
$$

\subsection{Numerical simulations}

Numerical simulations are performed using Matlab ${ }^{\circledR}$ ode45 integrator with $h=0.01$ and considering parameters: $\alpha=0.1, \beta_{1}=1, \beta_{3}=2, \delta_{1}=8.373, \rho_{1}=0.05, \rho_{2}=100$ and $\rho_{3}=200$ (Tusset et al., 2013). System (2.2) displays chaotic motions, as shown in Fig. 2.
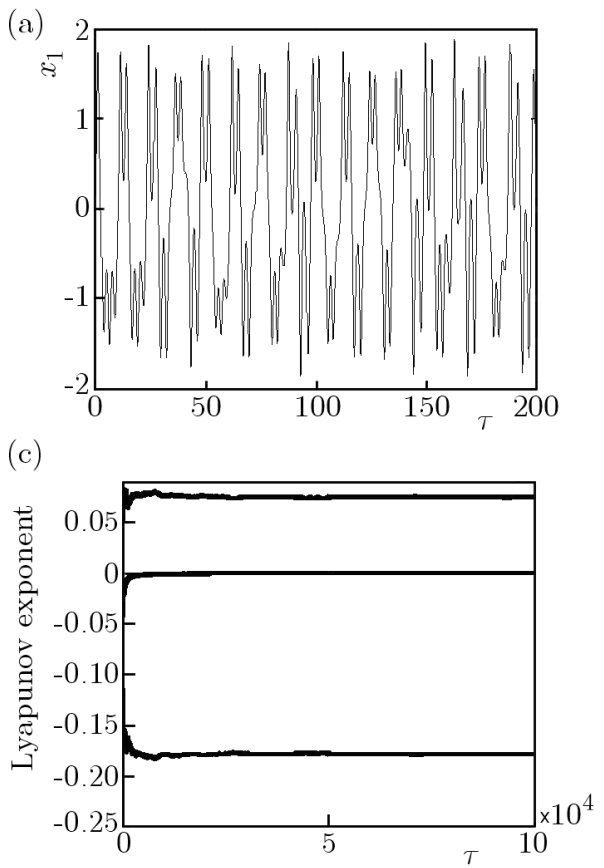

(b)

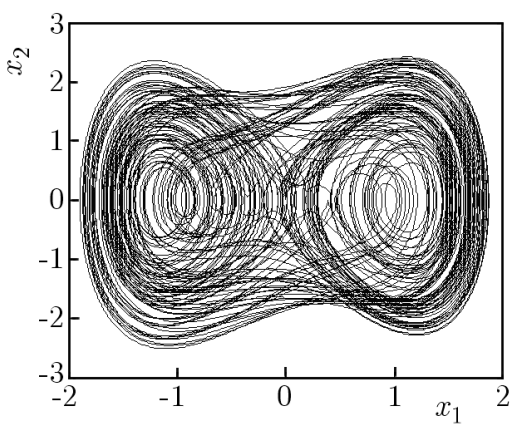

(d)

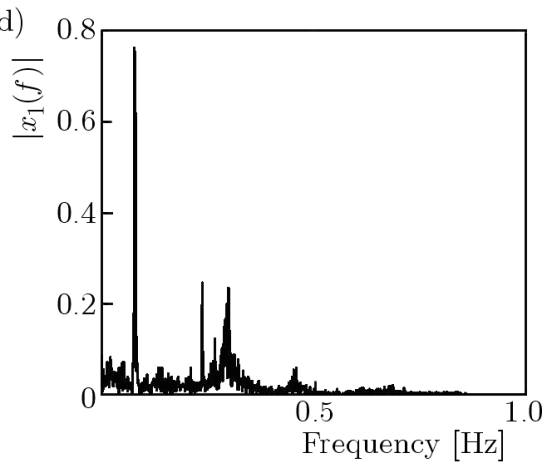

Fig. 2. (a) Displacement; (b) phase diagram; (c) Lyapunov exponent; (d) frequency spectrum

Computation of the first Lyapunov exponent $\lambda_{1}=0.075$ confirms chaotic behavior for the parameters used.

\section{Proposed active control}

Consider now the introduction of a controllable damper in the system, as shown in Fig. 3.

Introduction of the control $U$ leads system (2.2) to

$$
\begin{array}{ll}
x_{1}^{\prime}=x_{2} & x_{2}^{\prime}=-\alpha x_{2}+\beta_{1} x_{1}-\beta_{3} x_{1}^{3}+\delta_{1} \sin \left(x_{3}\right) x_{4}^{\prime}+\delta_{1} \cos \left(x_{3}\right) x_{4}^{2}+U \\
x_{3}^{\prime}=x_{4} & x_{4}^{\prime}=\rho_{1} \sin \left(x_{3}\right) x_{2}^{\prime}-\rho_{3} x_{4}+\rho_{2}
\end{array}
$$

where

$$
U=u^{*}+u
$$

$u^{*}$ will be the feedforward control and $u$ the feedback control. 

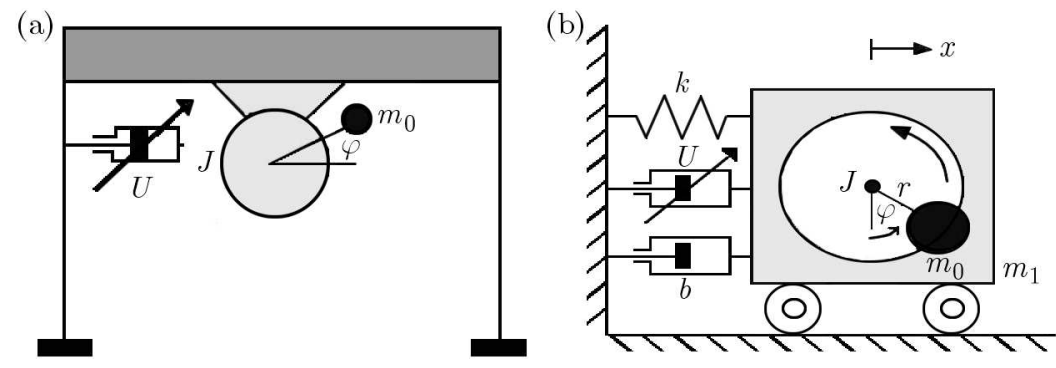

Fig. 3. (a) Portal frame with active control; (b) schematic oscillator with active control

Since the objective of this work is to control $x_{1}$ and $x_{2}$, the variables $x_{3}$ and $x_{4}$ will be considered only as disturbances of the system. Thus

$$
u^{*}=x_{2}^{* \prime}+\alpha x_{2}^{*}-\beta_{1} x_{1}^{*}+\beta_{3} x_{1}^{* 3}-\delta_{1} \sin \left(x_{3}\right) x_{4}^{\prime}-\delta_{1} \cos \left(x_{3}\right) x_{4}^{2}
$$

Substituting (3.3) into (3.1) and defining the deviation of the desired trajectory as

$$
\mathbf{y}=\left[\begin{array}{ll}
x_{1}-x_{1}^{*} & x_{2}-x_{2}^{*}
\end{array}\right]^{\mathrm{T}}
$$

we rewrite system (3.1) in matrix form

$$
\dot{\mathbf{y}}=\mathbf{A y}+\mathbf{G}\left(\mathbf{y}, \mathbf{x}^{*}\right)+\mathbf{B u}
$$

where

$$
\mathbf{A}=\left[\begin{array}{cc}
0 & 1 \\
\beta_{1} & -\alpha
\end{array}\right] \quad \mathbf{B}=\left[\begin{array}{l}
0 \\
1
\end{array}\right] \quad \mathbf{G}\left(y, x^{*}\right)=\left[\begin{array}{c}
0 \\
-\beta_{3}\left(y_{1}+x_{1}^{*}\right)^{3}+\beta_{3} x_{1}^{* 3}
\end{array}\right]
$$

The feedback control $\mathbf{u}$ can be found solving Eq. (3.6)

$$
\mathbf{u}=-\mathbf{R}^{-1} \mathbf{B}^{\mathrm{T}} \mathbf{P} \mathbf{y}
$$

According to Rafikov et al. (2008), if there are matrices $\mathbf{Q}$ and $\mathbf{R}$ (Q symmetric positive definite) such that

$$
\mathbf{Q}^{*}=\mathbf{Q}-\mathbf{G}^{\mathrm{T}}\left(\mathbf{y}, \mathrm{x}^{*}\right) \mathbf{P}-\mathbf{P G}\left(\mathbf{y}, \mathrm{x}^{*}\right)
$$

is positive definite, the matrix $\mathbf{G}$ restricted, then the control $\mathbf{u}$ is optimal and transfers the non-linear systems from any initial state to the final state $\mathbf{y}(\infty)=\mathbf{0}$

$$
J=\int_{0}^{\infty}\left(\mathbf{y}^{\mathrm{T}} \mathbf{Q}^{*} \mathbf{y}+\mathbf{u}^{\mathrm{T}} \mathbf{R} \mathbf{u}\right) d t
$$

The symmetric matrix $\mathbf{P}$ can be found from the Riccati algebraic equation

$$
\mathbf{P A}+\mathbf{A}^{\mathrm{T}} \mathbf{P}-\mathbf{P B R}^{-1} \mathbf{B}^{\mathrm{T}} \mathbf{P}+\mathbf{Q}=\mathbf{0}
$$

For the optimal control verification (3.6), function (3.7) is numerically calculated using

$$
L(t)=\mathbf{y}^{\mathrm{T}} \widetilde{\mathbf{Q}} \mathbf{y}
$$

The sufficient criterion to guarantee that control (3.8) is optimal is that $L(t)$ is positive definite (Rafikov et al., 2008). 


\subsection{Numerical simulations}

Let us define the desired trajectory as being a periodic orbit $x_{1}^{*}=0.01 \cos (\pi \tau)$. As can be seen, in Fig. 2d, the choice of this orbit allows the system to keep out of the resonance region and with a low amplitude value of the displacement. Defining matrices

$$
\mathbf{Q}=\left[\begin{array}{cc}
1000 & 0 \\
0 & 1000
\end{array}\right] \quad \mathbf{R}=[0.001]
$$

and solving the Riccati algebraic equation (3.9), we obtain the optimal feedback control (3.6)

$$
u=-1001\left(x_{1}-x_{1}^{*}\right)-1001.9\left(x_{2}-x_{2}^{*}\right)
$$

Substituting feedforward control (3.3) and the optimal feedback control (3.11) into (3.1), we will obtain trajectories shown in Fig. 4.
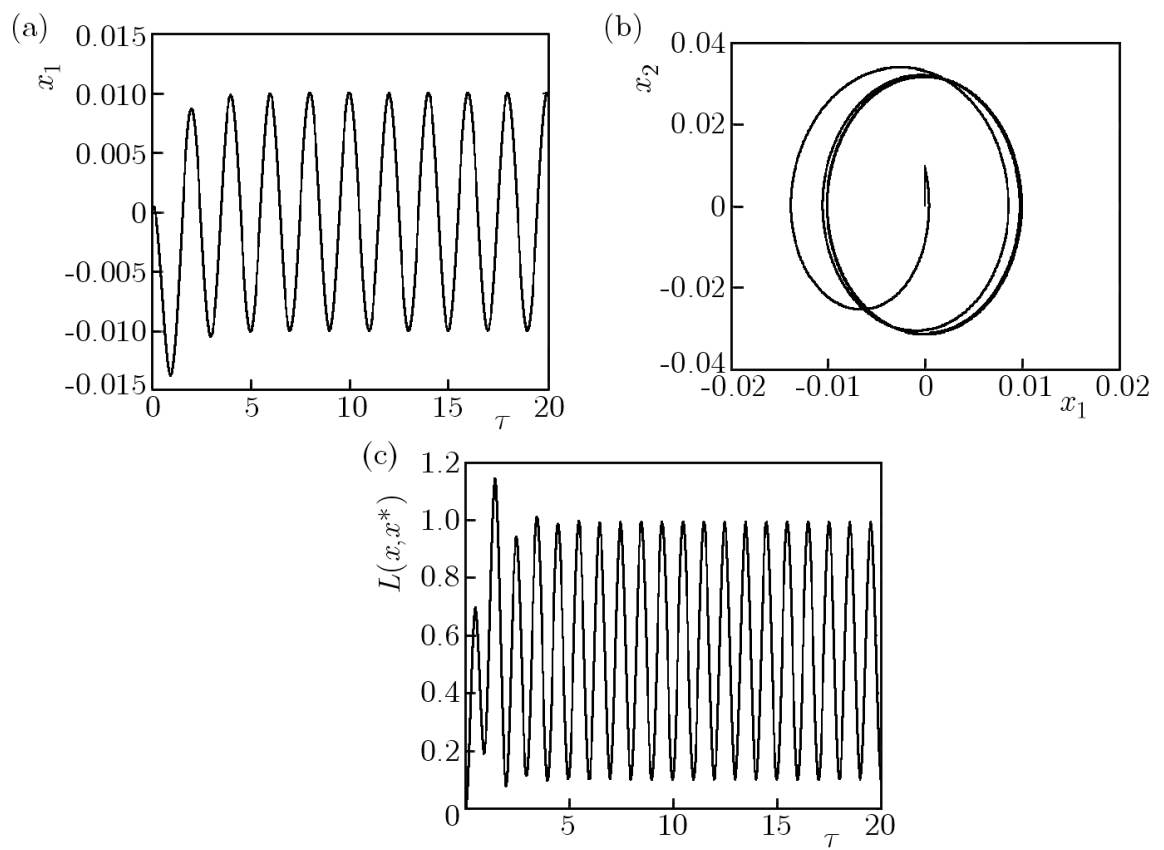

Fig. 4. (a) Displacement with active control; (b) phase portrait with active control; (c) $L(t)$ calculated in the optimal trajectory

As can be observed in Figs. 4a and 4b, the control is effective in reducing the displacement amplitude and frequency desired, and is optimal as shown in Fig. 4c.

\section{Proposed control by MR damper with hysteresis}

The MR damper has hysteresis effects due to a nonlinear friction mechanism. Many research efforts have been devoted to the modeling of this nonlinear behavior. An alternative is the LuGre friction model (Sakai et al., 2003) which was originally developed to describe nonlinear friction phenomena (Jimenez and Alvarez, 2002).

The friction mechanism is a phenomenon in which two surfaces make contact at a number of asperities at the microscopic level. In the modified LuGre friction model (Jimenez and Alvarez, 2002), this mechanism is expressed by the average behavior of the bristles. In (Sakai et al., 2003; Cetin et al., 2011), another MR damper model based on the LuGre model is described as

$$
\begin{aligned}
& F=\sigma_{a} z+\sigma_{0} z v+\sigma_{1} \dot{z}+\sigma_{2} \dot{x}+\sigma_{b} \dot{x} v \\
& \dot{z}=\dot{x}-\sigma_{0} a_{0}|\dot{x}| z
\end{aligned}
$$


where $F$ is the damping force, $v$ is the input voltage, $z(t)$ is the internal state variable $[\mathrm{m}], \dot{x}$ is the velocity of the damper piston $[\mathrm{m} / \mathrm{s}], \sigma_{0}$ is the stiffness of $z(t)$ influenced by $v[\mathrm{~N} /(\mathrm{mV})], \sigma_{1}$ is the damping coefficient of $z(t)[\mathrm{Ns} / \mathrm{m}], \sigma_{2}$ is the viscous damping coefficient $[\mathrm{Ns} / \mathrm{m}], \sigma_{a}$ is the stiffness of $z(t)[\mathrm{N} / \mathrm{m}], \sigma_{b}$ is the viscous damping coefficient influenced by $v[\mathrm{Ns} /(\mathrm{mV})], a_{0}$ is the constant value $[\mathrm{V} / \mathrm{N}]$.

As can be seen in equation $(4.1)_{1}$, the LuGre model has a parameter that represents the voltage applied to the coil of the damper $v$. This parameter allows one to control the force of the MR damper through the control of the voltage $v$ making the LuGre model the most suitable for the active control system.

\subsection{Control of oscillations using an active MR damper}

For numerical simulations we will consider $\dot{x}$ in equations (4.1) as $\dot{x}=-0.01 \pi \sin (\pi \tau)$, and the parameters: $\sigma_{0}=8 \cdot 10^{5}, \sigma_{1}=1.6 \cdot 10^{3}, \sigma_{2}=1.5 \cdot 10^{2}, \sigma_{a}=4 \cdot 10^{5}, \sigma_{b}=8 \cdot 10^{2}$ and $a_{0}=3 \cdot 10^{-3}$ (Sakai et al., 2003). In Fig. 5, one can observe the force the damper MR $(4.1)_{1}$.
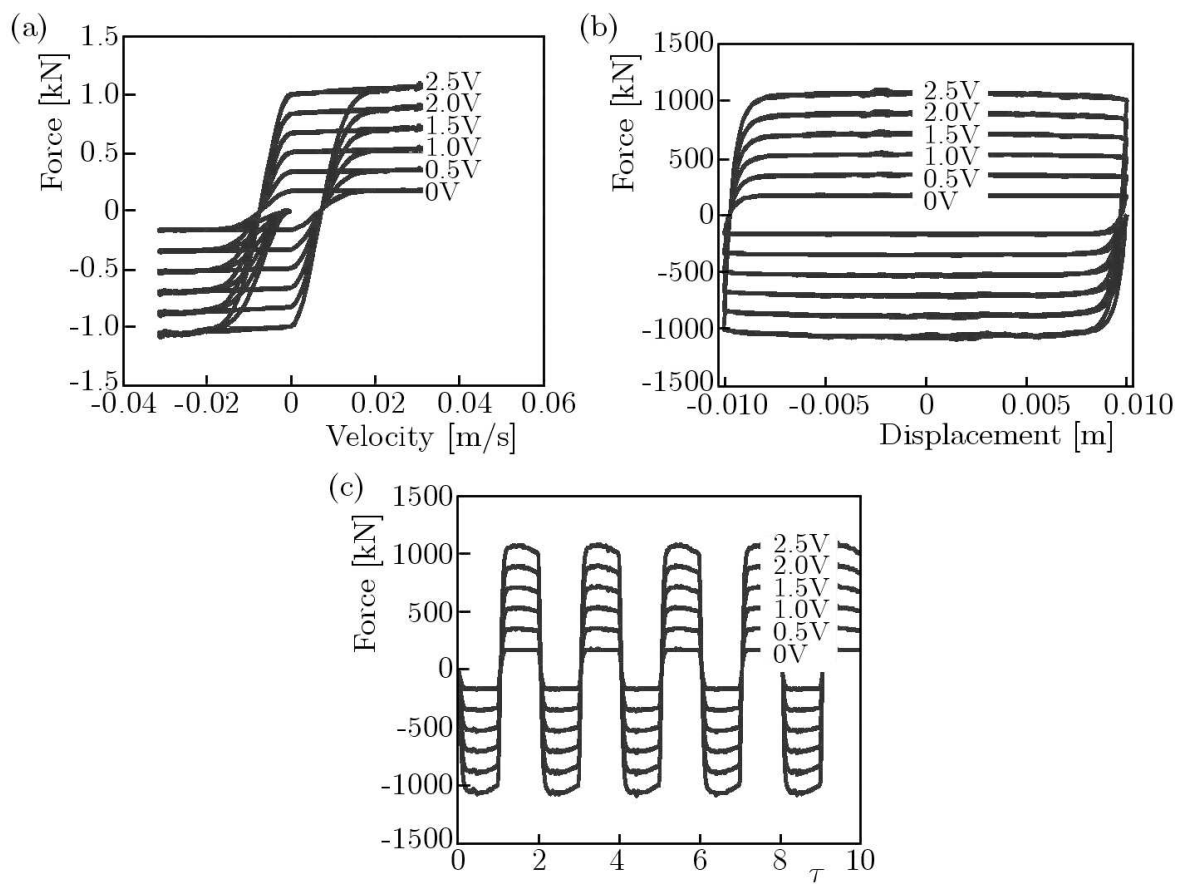

Fig. 5. Characteristics of the MR damper as a function of voltage: (a) force vs. velocity, (b) force vs. displacement, (c) force vs. time

The MR damper semi-active system and the damping force can be controlled by controlling the applied voltage in the damper coil. According to Tusset et al. (2013), one can numerically determine the voltage $v$ required for the force of the MR damper $F(4.1)_{1}$ to coincide with the desired control force $U,(3.2)$, obtained from the control strategy.

Considering $F=U$, the voltage to be applied can be determined by solving the following function

$$
\Gamma(v)=\sigma_{a} z+\sigma_{0} z v+\sigma_{1} \dot{z}+\sigma_{2} \dot{x}+\sigma_{b} \dot{x} v-U
$$

Through Eq. (4.2), we can determine the voltage being applied to the control system considering the force estimated by the control method, such as control proposed in this paper. In Fig. 6, we can see the force applied to control the oscillations. 


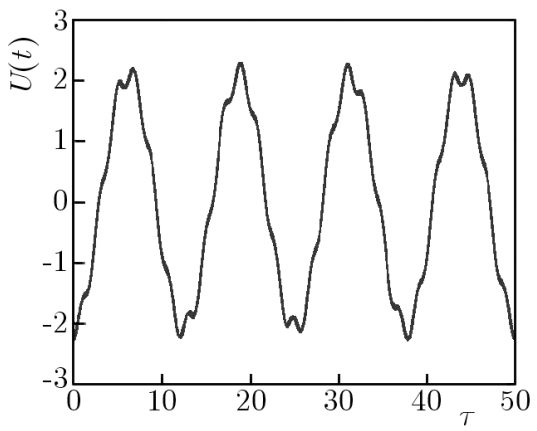

Fig. 6. Force used to control the non-ideal system

Considering forces $U$ (Fig. 6) and $F$ (Fig. 5) normalized, the voltage to be applied is determined by solving numerically function (4.2). In Fig. 7, we see the values of $z, \dot{z}$ and $\dot{x}$ used in equation (4.2).
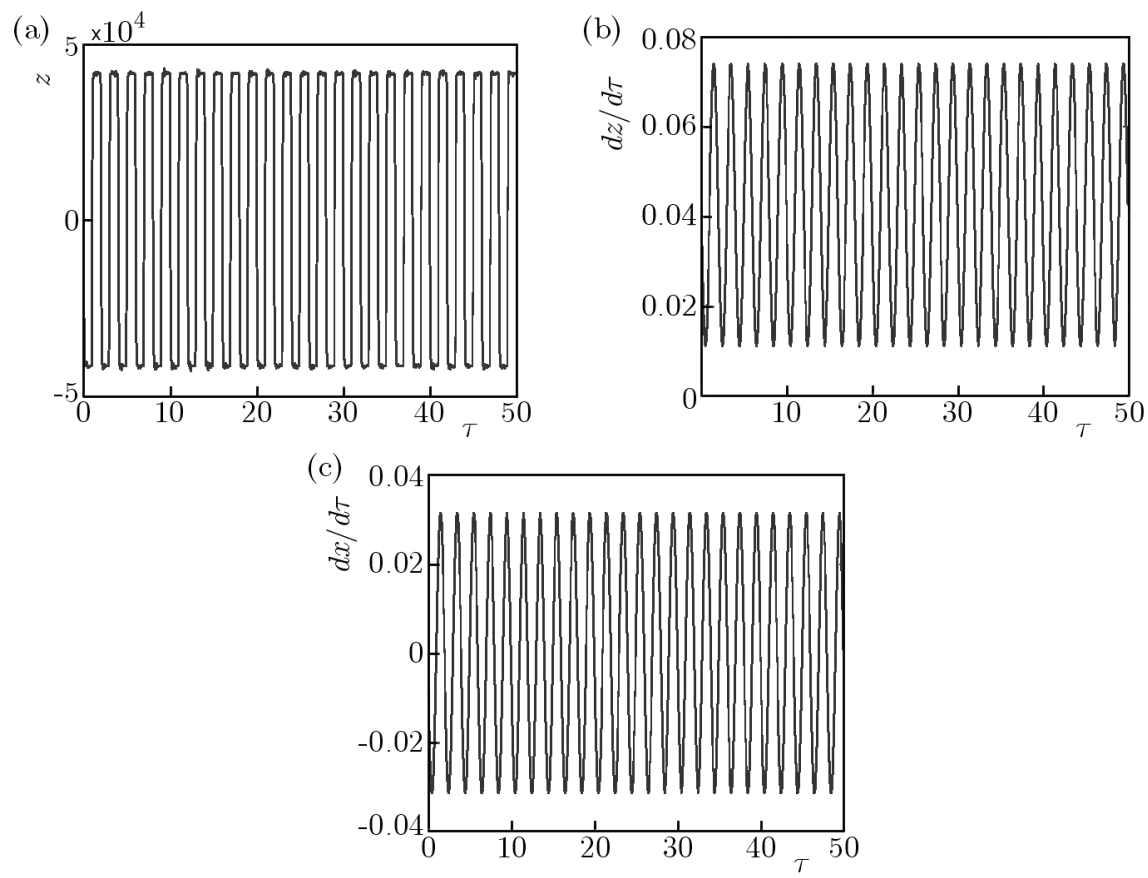

Fig. 7. (a) Internal state variable $z(\tau)$; (b) derivative of internal state variable $\dot{z}(\tau)$; (c) velocity of the piston of the damper $\dot{x}(\tau)$

In Fig. 8, we can see the voltage control estimated considering Eq. (4.2) and values from Fig. 7.

Considering these results, we can observe that the proposed methodology allowed the control of the voltage (Fig. 8) considering the force $U$ in Fig. 6 and Eq. (4.2).

\subsection{Control of oscillations using a passive MR damper}

The objective of introducing a passive MR damper to the portal frame structure shown in Fig. 9 is to control the displacement of the portal frame in the same scale considered in the proposed active control.

Next, we will consider the introduction of a passive MR damper $U_{p}$ into system (2.2)

$$
\begin{array}{ll}
x_{1}^{\prime}=x_{2} & x_{2}^{\prime}=-\alpha x_{2}+\beta_{1} x_{1}-\beta_{3} x_{1}^{3}+\delta_{1} \sin \left(x_{3}\right) x_{4}^{\prime}+\delta_{1} \cos \left(x_{3}\right) x_{4}^{2}-U_{p} \\
x_{3}^{\prime}=x_{4} & x_{4}^{\prime}=\rho_{1} \sin \left(x_{3}\right) x_{2}^{\prime}-\rho_{3} x_{4}+\rho_{2}
\end{array}
$$




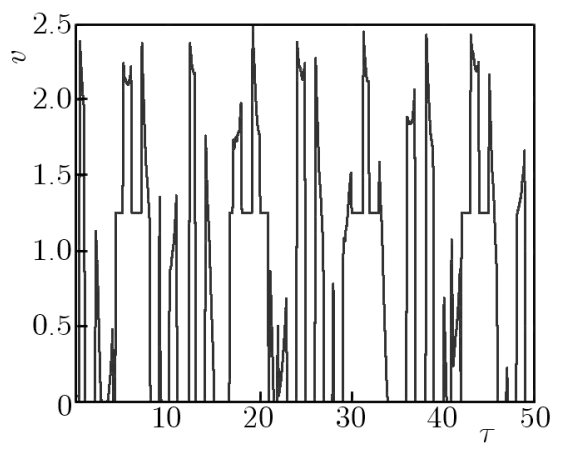

Fig. 8. Voltage used to control the non-ideal system
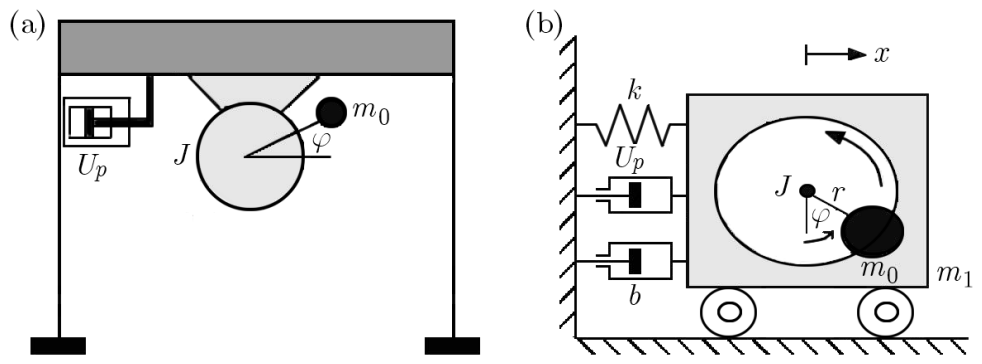

Fig. 9. (a) Portal frame with passive control; (b) schematic oscillator with passive control

where

$$
U_{p}=\sigma_{a} z+\sigma_{0} z v+\sigma_{1} \dot{z}+\sigma_{2} \dot{x}+\sigma_{b} \dot{x} v
$$

and $z$ is obtained by solving Eq. (4.1) 2 .

In Figs. 10-13, we can observe motions of the portal frame for different constant voltages $v$ applied to the MR damper, (4.4).
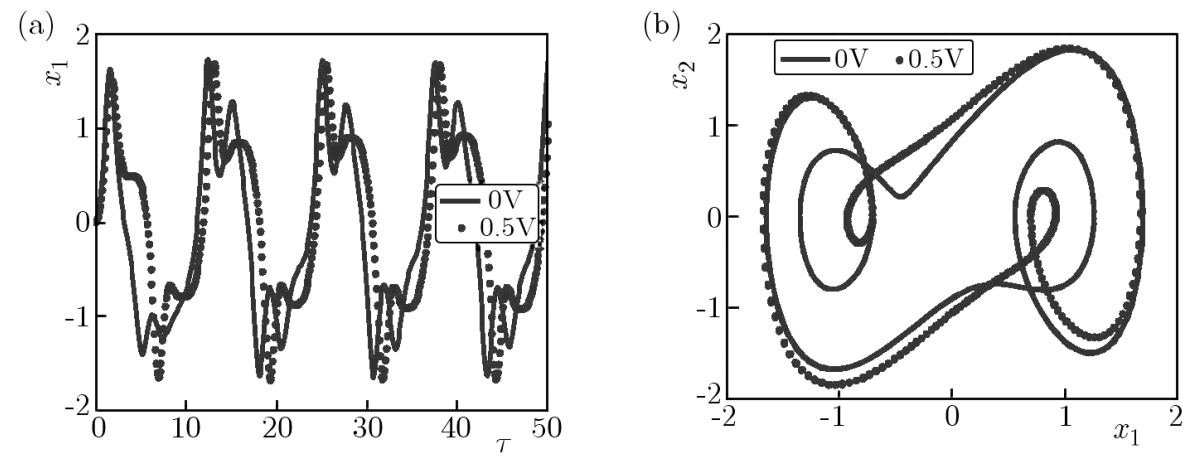

Fig. 10. Voltage $\nu=0 \mathrm{~V}$ and $\nu=0.5 \mathrm{~V}$ : (a) displacement, (b) phase portrait

We can observe that as we increase the value of voltage, the amplitude of the displacement reduces and shifts the equilibrium point of origin. We also observe that for $\nu=2.5 \mathrm{~V}$ the amplitudes are smaller than those obtained with the active damper. For $\nu=2.3808 \mathrm{~V}$ we have the same displacement amplitude for the active damper and passive damping, as can be seen in Fig. 14.

As it can be seen in Fig. 14, it is possible to maintain the system on periodic orbits using the MR damper energized with $\nu=2.3808 \mathrm{~V}$. One factor which compromises this control strategy is that the system stabilizes after a long time $\tau>113.5$, and it is not recommended to keep the coil of the MR damper energized continuously for long periods, as required by the proposed active control (Fig. 8). 
(a)

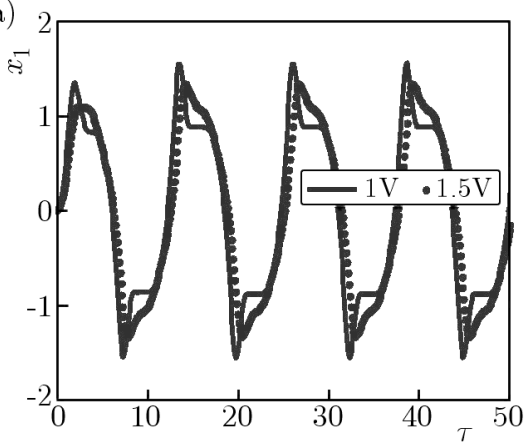

(b)

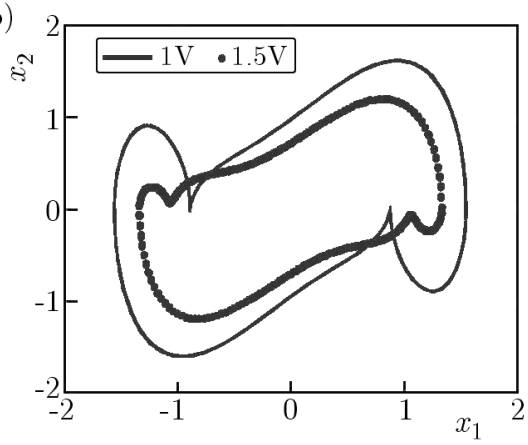

Fig. 11. Voltage $\nu=1 \mathrm{~V}$ and $\nu=1.5 \mathrm{~V}$ : (a) displacement, (b) hase portrait
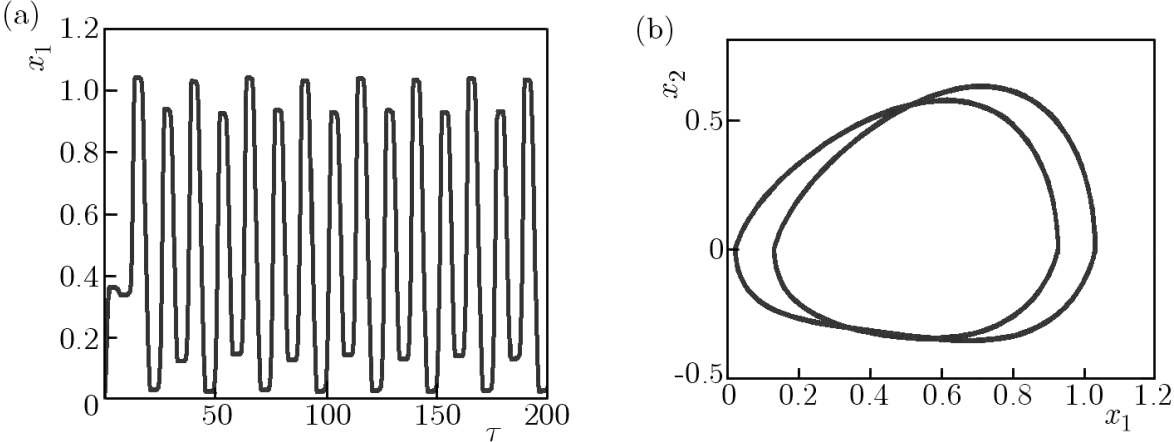

Fig. 12. Voltage $\nu=2 \mathrm{~V}$ : (a) displacement, (b) phase portrait

(a)

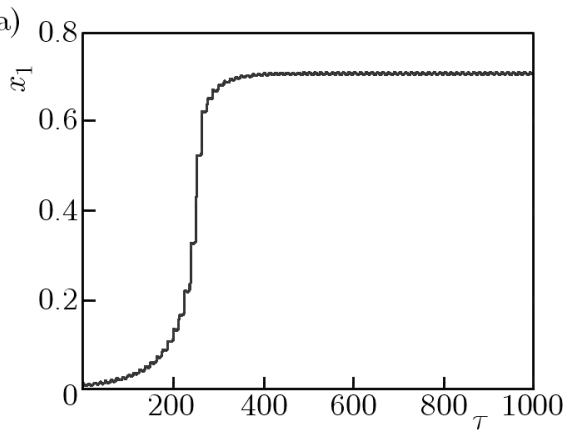

(b)

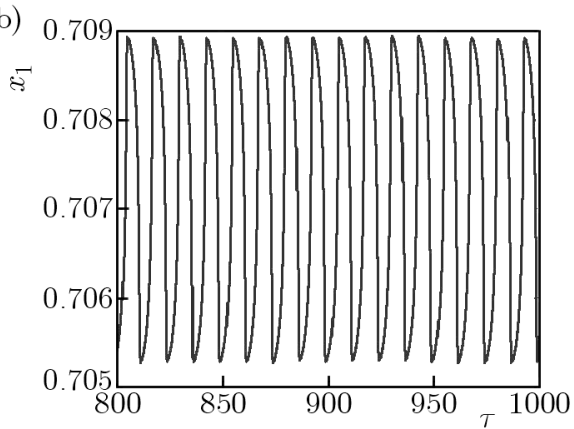

(c)

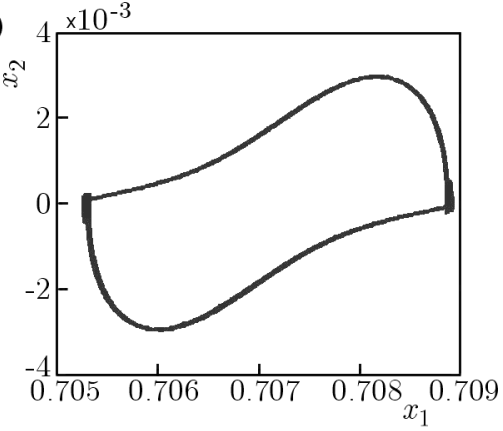

Fig. 13. Voltage $\nu=2.5 \mathrm{~V}$ : (a) displacement for $0 \leqslant \tau \leqslant 1000$, (b) displacement for $800 \leqslant \tau \leqslant 1000$, (c) phase portrait 

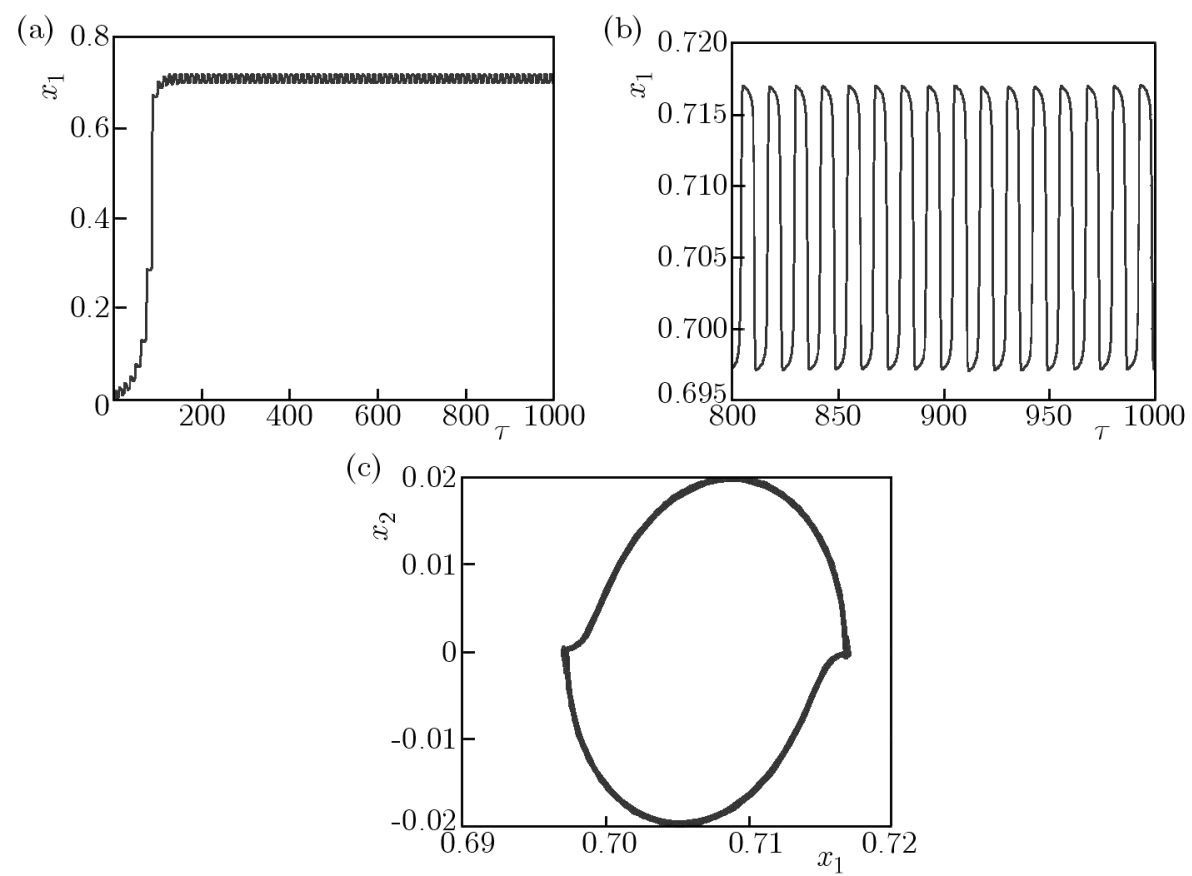

Fig. 14. Voltage $\nu=2.3808 \mathrm{~V}$ : (a) displacement for $0 \leqslant \tau \leqslant 1000$, (b) displacement for $800 \leqslant \tau \leqslant 1000$, (c) phase portrait

\section{Active control with uncertainties}

The parameters used in the control strategy are obtained from a certain data set. The data set provides parametric errors due to measurement errors or model uncertainties. To consider the effect of parameter uncertainties on the performance of the controller, the parameters used in the proposed control will be considered having a random error of $\pm 20 \%$ (Balthazar et al., 2014).

In order to consider the effect of parameter uncertainties on the performance of the controller, the real unknown parameters of the system are supposed to be as follows: $\widehat{\alpha}=0.08+0.04 r(t)$, $\widehat{\beta}_{1}=0.8+0.4 r(t), \widehat{\beta}_{3}=1.6+0.8 r(t), \widehat{\delta}_{1}=6.6984+3.3492 r(t), \widehat{\rho}_{1}=0.04+0.02 r(t)$, $\widehat{\rho}_{2}=80+40 r(t)$ and $\hat{\rho}_{3}=160+80 r(t)$, where $r(t)$ are normally distributed random functions. To analyze the sensitivity of the error, we consider

$$
\left[\begin{array}{l}
e_{1} \\
e_{2}
\end{array}\right]=\left[\begin{array}{l}
x_{1}-\widehat{x}_{1} \\
x_{2}-\widehat{x}_{2}
\end{array}\right]
$$

where $x_{i}$ is obtained for the control without parametric errors and $\widehat{x}_{i}$ is obtained for the control with the parametric error $(i=1,2)$.
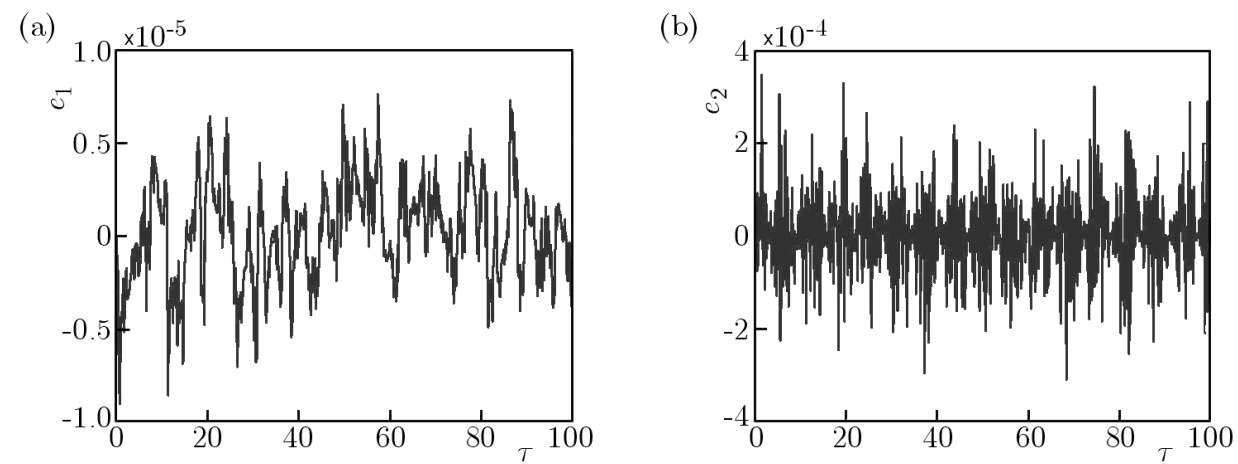

Fig. 15. Error of uncertainty in parameters: (a) $x_{1}-\widehat{x}_{1}$, (b) $x_{2}-\widehat{x}_{2}$ 
In Fig. 15, we can observe the sensitivity of the proposed active control with parametric errors.

As can be observe in Fig. 15, the proposed active control is somewhat sensitive to parametric errors being under $10^{-4}$.

\section{Conclusions}

As can be seen in Figs. 4a and 4b, with the proposed association of feedforward control $u^{*}$ (3.3) and feedback $u$ (3.11), it is possible to control the oscillations of the studied portal frame structure under non-ideal loading into periodic orbits, and the control is optimal (Fig. 4c). Our model of the MR damper force (4.2) allows us to determine the electrical current to be applied to the dampers, as shown in Fig. 8. Regarding the use of the passive control, it is possible to observe that to keep the system in a periodic displaced orbit, the origin must be changed. Thus, the active control is more indicated, as it keeps the oscillations around the origin. With respect to the parametric sensitivity, the active control proved to be less sensitive than the passive control.

With the results obtained, we can conclude that the proposed active control using a MR damper is more appropriate than the use of a passive MR damper.

Acknowledgments

The authors acknowledge the financial support by FAPESP and CNPq (grant: 420026/2013-4 and grant: 484729/2013-6), both Brazilian research funding agencies.

\section{References}

1. Balthazar J.M., Tusset A.M., Bueno A.M., 2014, TM-AFM nonlinear motion control with robustness analysis to parametric errors in the control signal determination, Journal of Theoretical and Applied Mechanics, 52, 1, 93-106

2. Bolla M., Balthazar J.M., Felix J.L.P., Mook D.T., 2007, On an approximate analytical solution to a nonlinear vibrating problem, excited by a non-ideal motor, Nonlinear Dynamics, 50, $841-847$

3. Castão K.A.L., Goes L.C.S., Balthazar J.M., 2010, A note on the attenuation of the sommerfeld effect of a non-ideal system taking into account a MR damper and the complete model of a DC motor, Journal of Vibration and Control, 17, 7, 1112-1118

4. Cetin S., Zergeroglu E., Sivrioglu S., Yuksek I., 2011, A new semiactive nonlinear adaptive controller for structures using MR damper: Design and experimental validation, Nonlinear Dynamics, 66, 4, 731-743

5. DJanan A.A.N., NBendjo B.R.N., WoAfo P., 2013, Electromechanical control of vibration on a plate submitted to a non-ideal excitation, Mechanics Research Communications, 54, 72-82

6. Dutta S., Chakraborty G., 2014, Performance analysis of nonlinear vibration isolator with magneto-rheological damper, Journal of Sound and Vibration, 333, 5097-5114

7. Dyke S.J., Spencer B.F. Jr., Sain M.K., Carlson J.D., 1996, Modeling and control of magnetorheological dampers for seismic response reduction, Smart Materials and Structures, 5, $565-575$

8. Jimenez R., Alvarez L., 2002, Real time identification of structures with magnetorheological dampers, Proceedings of the 41st IEEE Conference on Decision and Control, 1017-1022

9. Jimenez R., Alvarez L., 2005, LuGre friction model for a magnetorheological damper, Structural Control and Health Monitoring, 12, 91-116 
10. Kasemi B., Muthalif A.G.A., Rashid M.M., Fathima S., 2012, Fuzzy-PID Controller for semi-active vibration control using magnetorheological fluid damper, Procedia Engineering, 41, $1221-1227$

11. Piccirillo V., Tusset A.M., Balthazar J.M., 2014, Dynamical jump attenuation in a non-ideal system through magneto rheological damper, Journal of Theoretical and Applied Mechanics, $\mathbf{5 2}, 2,595-604$

12. Rafikov M., Balthazar J.M., Tusset A.M., 2008, An optimal linear control design for nonlinear systems, Journal of the Brazilian Society of Mechanical Sciences and Engineering, 30, 279-284

13. Rafikov M., Balthazar J.M., 2004, On an optimal control design for Rössler system, Physics Letters A, 333, 241-245

14. Sakai C., Ohmori H., SAno A., 2003, Modeling of MR damper with hysteresis for adaptive vibration control, Proceedings of the 42nd IEEE Conference on Decision and Control, 3840-3845

15. Samantaray A.K., Dasgupta S.S., Bhattacharyya R., 2010, Sommerfeld effect in rotationally symmetric planar dynamical systems, International Journal of Engineering Science, 48, 21-36

16. Spencer Jr. B.F., Dyke S.J., Sain M.K., Carlson J.D., 1997, Phenomenological model of a magnetorheological damper, ASCE Journal of Engineering Mechanics, 3, 230-238

17. Terasawa T., Sakai C., Ohmori H., Sano A., 2004, Adaptive identification of MR damper for vibration control, Proceedings of the 43rd IEEE Conference on Decision and Control, 14-17

18. Tusset A.M., Balthazar J.M., 2013, On the chaotic suppression of both ideal and non-ideal Duffing based vibrating systems, using a magnetorheological damper, Differential Equations and Dynamical Systems, 21, 105-121

19. Tusset A.M., Balthazar J.M., Felix J.L.P., 2013, On elimination of chaotic behavior in a non-ideal portal frame structural system, using both passive and active controls, Journal of Vibration and Control, 19, 803-813

20. Tusset A.M., Balthazar J.M., Chavarette F.R., Felix J.L.P., 2012, On energy transfer phenomena, in a nonlinear ideal and nonideal essential vibrating systems, coupled to a (MR) magneto-rheological damper, Nonlinear Dynamics, 69, 1859-1880

21. Tusset A.M., Rafikov M., Balthazar J.M., 2009, Intelligent controller design for magnetorheological damper based on quarter-car model, Journal of Vibration and Control, 15, 1907-1920

Manuscript received May 9, 2014; accepted for print February 10, 2015 